\title{
Use of Ultra High Pressure Homogenization to sterilize grape must
}

\author{
A. Morata ${ }^{1, a}$, I. Loira ${ }^{1}$, M.A. Bañuelos ${ }^{2}$, A. Puig-Pujol ${ }^{3}$, B. Guamis ${ }^{4}$, C. González ${ }^{1}$, and J.A. Suárez-Lepe ${ }^{1}$ \\ 1 enotecUPM, Chemistry and Food Technology Department, ETSIAAB, Universidad Politécnica de Madrid, Avenida Puerta de Hierro \\ 2-4, 28040 Madrid, Spain \\ ${ }^{2}$ Dept. Biotecnología-Biología Vegetal, ETSIAAB, Universidad Politécnica de Madrid, Spain \\ ${ }^{3}$ Enological Research Department, Institut of Agrifood Research and Technology, Catalan Institute of Vine and Wine (IRTA-INCAVI), \\ Plaça Àgora, 2, 08720 Vilafranca del Penedès, Spain \\ ${ }^{4}$ Centre d'Innovació, Recerca i Transferència en Tecnologia dels Aliments (CIRTTA), TECNIO, XaRTA, Departament de Ciència \\ Animal i dels Aliments, Facultat de Veterinària, Universitat Autònoma de Barcelona, 08193 Bellaterra, Spain
}

\begin{abstract}
Ultra-High Pressure Homogenization Sterilization (UHPHS) allows the sterilization of fluid foods at low temperatures or even in refrigeration. UHPHS is a continuous technique that allows to process $10,000 \mathrm{~L} / \mathrm{h}$ with a single pump working at $300 \mathrm{MPa}$ with an imbalance of $1 \mathrm{MPa}$ (Ypsicon EP2409583). During the process, fluid temperature increases less than $5^{\circ} \mathrm{C}$. The technique is sensory gentle not affecting molecules formed by covalent bonds so aroma and pigments are unaltered. During the process of white musts, the complete elimination of yeasts, bacteria and spores can be achieved and the must may remain unfermented for several months-years if stored in amicrobic conditions. The technique is also effective in the destruction of polyphenol oxidase (PPO) enzymes. Final size particle is $100-300 \mathrm{~nm}$ allowing to increase the availability of yeast assimilable nitrogen and opens the opportunity to nano-encapsulate flavours. Destruction of PPOs and elimination of microorganisms help to reduce sulphites in wines. UHPHS facilitates the use of new biotechnologies such as the use of non-Saccharomyces yeasts and yeast-bacteria co-inoculations by enabling a better implantation of the starters in absence of competition with wild grape microorganisms.
\end{abstract}

\section{Introduction}

Ultra-High Pressure Homogenization Sterilization (UHPHS) is a non-thermal emergent continuous technique [1], based in the processing of a fluid at $300 \mathrm{MPa}$ or higher (Fig. 1a). UHPHS systems able to process food fluids at a flow of $10,000 \mathrm{~L} / \mathrm{h}$ are currently available (Ypsicon [2], EP2409583 [3], Fig. 1b). This flow can be increased with multi-modular units in which several UHPH pumps are working in parallel. UHPHS is currently under consideration by OIV to be included in the approved practices. In 2019, it is at step 5 (OENO-MICRO 16-594B Et5). This technique not only produces the elimination of yeast and bacteria but also spores. The main mechanisms of inactivation are extreme impact and shear forces when the fluid that is pumped at $300 \mathrm{MPa}$ is depressurized to atmospheric pressure (Fig. 1c) through a specifically designed valve. In the valve the intense forces increase the temperature until $98^{\circ} \mathrm{C}$ but just during less than $0.02 \mathrm{~s}$ [4], the subsequent expansion chills the fluid producing a global increase of temperature lower than $5^{\circ} \mathrm{C}$. UHPHS cannot be considered a thermal technique because conventional thermal sterilization needs to eliminate spores $121^{\circ} \mathrm{C}$ 15 minutes or alternatively $138^{\circ} \mathrm{C}-4 \mathrm{~s}$. When UHPHS process is used, the conditions are $98^{\circ} \mathrm{C}-0.02 \mathrm{~s}$. That produces a gentle treatment of musts in terms of sensory quality, remaining molecules with sensory repercussion (anthocyanins and aroma compounds) unaffected [4]. In general terms, small molecules formed by covalent bonds, remain unaffected and Maillard reactions or thermal markers compounds such as furfural or 5-hydroxymethil furfural are not formed.

Fluids with colloidal particles can be processed by UHPHS, but it is necessary a size lower than $500 \mu \mathrm{m}$. This produces the current inapplicability to crushed grapes to continuously produce red wines, even when this technique will increase highly the extraction of phenolic compounds. We are working in new grape pre-treatments to facilitate the subsequent application of UHPHS for red must maceration.

The power consumption of this technique is reduced and can be estimated as lower than $30 \%$ of the consumption in discontinuous high hydrostatic pressure (HHP). Moreover, is a clean and sustainable technology with low water requirement and low levels of effluents.

\section{UHPHS effectivity and properties}

\subsection{Elimination of microorganisms and sterilization}

As previously described, the main inactivation mechanisms are the high impact and shears forces produced in the depressurization valve. Therefore, inactivation mechanism of UHPHS is completely different to discontinuous HHP in which pressure is the main inactivation mechanism. In HHP the energy is enough to eliminate wine yeasts at 

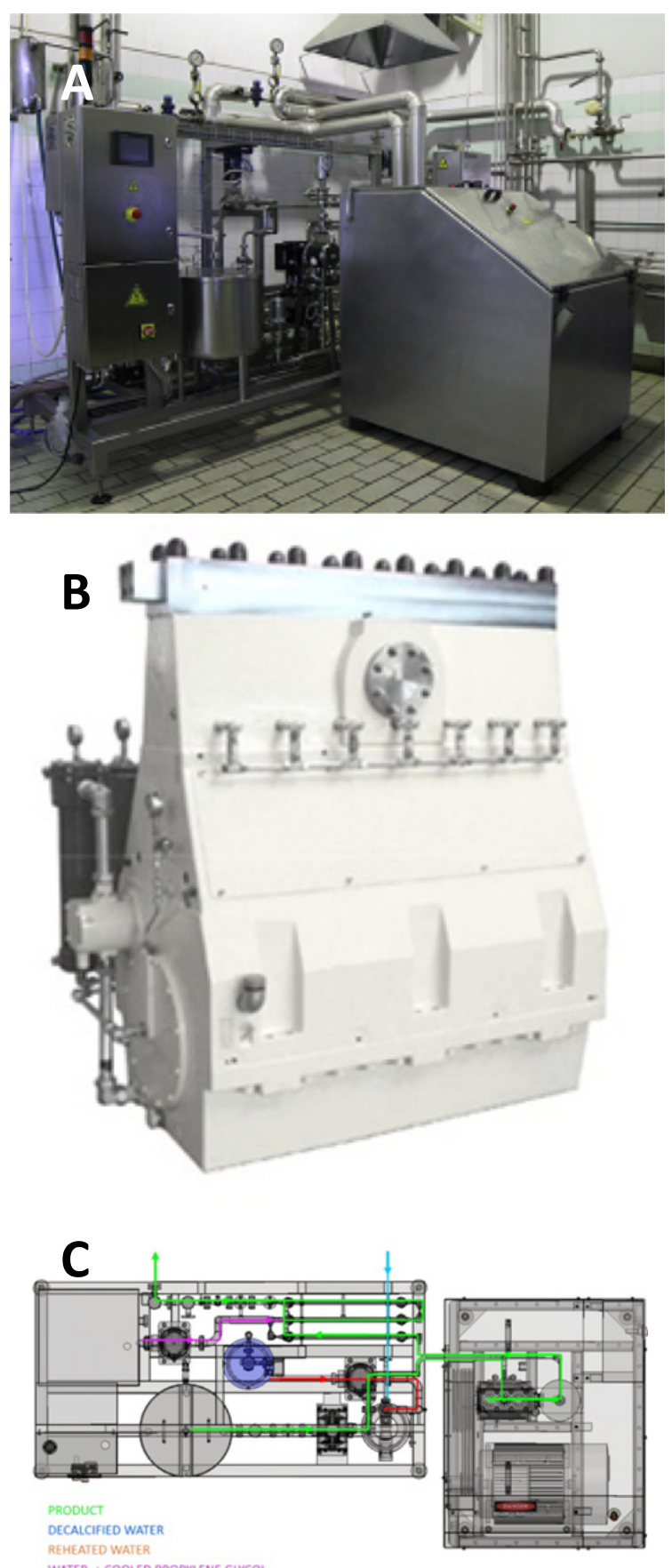

Figure 1. A. UHPHS device. B. UHPH pump 10,000 L/h. C. Sections with fluid flow (Ypsicon).

300-400 MPa/10 minutes, including Saccharomyces and non-Saccharomyces species [5]. However, bacteria are strongly reduced, but remain at populations around 1-log $\mathrm{CFU} / \mathrm{mL}$ even when $550 \mathrm{MPa} / 10$ minutes treatments are used [5].

In UHPH, the extremely intense impact and shear forces produce the disaggregation of whatever colloidal particle or microorganism to small fragments lower than $300 \mathrm{~nm}$. The effect in the particles through the valve can be compared with the energy of a reaction plane flying at 3 -fold sound speed crashing with a mountain.

When UHPHS is used on grape musts, yeasts and bacteria are eliminated, also spores, and musts remain unfermented during several months-years. Wild yeast

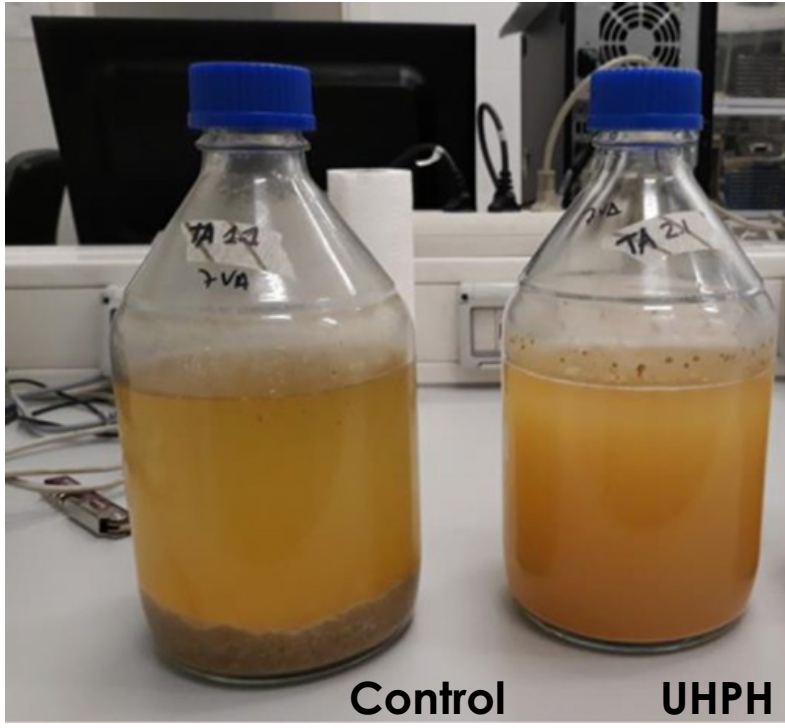

Figure 2. Wine after fermentation from must unprocessed or UHPHS treated.

populations of 6-log CFU/mL (both Saccharomyces and non-Saccharomyces) were completely eliminated when the must was treated with UHPH. When the must was inoculated with a starter, just the inoculated strain was detected at the end of fermentation, with complete absence of wild non-Saccharomyces yeasts [4]. Same results were observed for wild bacteria populations of 4-log $\mathrm{CFU} / \mathrm{mL}$. Moreover, bacteria remain undetected after must fermentation [4].

\subsection{Sensory impact and colloidal effects}

The fragmentation of colloidal particles during UHPHS process produces really stable colloidal structures, because all particles are depolymerized to $100-300 \mathrm{~nm}$ (Fig. 2).

In Fig. 2, after fermentation of the must only clarified by cold, it is possible to see that most of the colloidal particles settled in the bottom of the flask. However, the low molecular size in the UHPHS must keep it stable even after fermentation.

From a sensory point of view, musts keep varietal smells, and after fermentation, fruity aroma is perceived and high levels of esters can be measured [4]. Colour also remains unaffected with absence of caramelization or oxidative hints.

\section{Effects on enzymes, $\mathrm{SO}_{2}$ levels and new biotechnologies}

UHPHS is able to control oxidative enzymes such as polyphenol oxidases (PPOs), probably by the denaturalization of the ternary structure. When PPO activity has been measured before and after UHPHS treatment, a significant reduction of $90 \%$ was observed [4].

The full elimination of bacteria and wild nonSaccharomyces yeasts together with the elimination of oxidative enzymes makes UHPHS as an interesting technique to minimize $\mathrm{SO}_{2}$ in musts.

In current oenology, the use of non-Saccharomyces yeast is a powerful tool to modulate wine flavour, 
however, most of these species are low competitive with Saccharomyces cerevisiae because of their low fermentative power or slow fermentation kinetics. So, it is difficult to implant non-Saccharomyces yeasts in oenological conditions. Must sterilization by UHPHS helps to overcome this main drawback of the new technologies. Similar effects can be obtained when yeastbacteria inoculations (co-inoculation) are desired to reduce fermentation time. UHPHS treated musts facilitate the use of bacteria-yeast starters.

\section{Conclusions}

UHPHS is and effective, sustainable, and eco-friendly technique that allows $\mathrm{SO}_{2}$ reduction and the application of new fermentation biotechnologies.

\section{References}

[1] A. Morata, I. Loira, R. Vejarano, C. González, M.J. Callejo, J.A. Suárez-Lepe. Trends in Food Sci. Technol. 67, 36 (2017), https://doi. org/10.1016/j.tifs.2017.06.014

[2] Ypsicon (2018), Ultra high pressure homogenization/sterilization. Retrieved from http://www.ypsicon.com

[3] European Patent UHPHS. EP2409583. Ypsicon

[4] I. Loira, A. Morata, M.A. Bañuelos, A. Puig-Pujol, B. Guamis, C. González, J. A. Suárez-Lepe, Innovative Food Sci. Emerging Technol. 50, 50 (2018)

[5] A. Morata, I. Loira, R. Vejarano, M.A. Bañuelos, P.D. Sanz, L. Otero, J.A. Suárez-Lepe, Food and Bioprocess Technol. 8, 277 (2015) 